\title{
The effect of planar constraint on the definition of the wrist axes of rotation
}

Submitted as a Short Communication

\author{
Oluwalogbon O Akinnola, MEng ${ }^{1}$ \\ Vasiliki Vardakastani, $\mathrm{PhD}^{1}$ \\ Angela E Kedgley, $\mathrm{PhD}^{1 *}$
}

${ }^{1}$ Department of Bioengineering, Imperial College London, London, United Kingdom

Email addresses:

OOA: o.akinnola16@imperial.ac.uk

VV: v.vardakastani13@imperial.ac.uk

AEK: akedgley@imperial.ac.uk

* Corresponding author:

Angela E Kedgley

Department of Bioengineering

Imperial College London

South Kensington Campus

London SW7 2AZ

Phone: +44 (0) 2075940747 


\section{Abstract}

Instantaneous helical axes (IHAs) and screw displacement axes (SDAs) are commonly used to investigate joint functional axes of rotation. In the wrist, these have often been obtained through in vitro motion analysis. These definitions are then employed for in vivo applications, such as the design of implants or the development of musculoskeletal models. However, functional unguided joint motions are, by definition, affected by the activity of muscles. Previously published data has disagreed on the relative position and orientation of the two primary axes of rotation of the wrist, i.e. the radioulnar deviation (RUD) axes with respect to the flexion-extension (FE) axis. An in vivo study comparing the FE and RUD IHAs and SDAs of guided motions, to replicate in vitro conditions, and unguided motions of 23 healthy participants was conducted using optical motion capture. Guided motions were performed with the hand and forearm flush against a flat surface. The relative position and orientation of the RUD SDAs with respect to the FE SDAs differed $(p=0.019, p=0.001)$ between unguided FE and guided RUD $\left(0.1 \pm 4.3 \mathrm{~mm}, 93.5 \pm 16.0^{\circ}\right)$ and guided FE and RUD $(1.6 \pm 4.0 \mathrm{~mm}, 107.8$ $\left.\pm 17.7^{\circ}\right)$. This indicates that the use of different constraints, and not physiological differences, is the cause for differences in the relative positions and orientations of the FE and RUD axes in the literature. Thus, the practice of using in vitro definitions of the axes of rotation of the wrist for in vivo applications, especially involving $\mathrm{FE}$, may be inappropriate and care must be taken to account for any constraint on wrist motion. It is recommended that investigators define the axes of rotation specifically for their study or refer to literature featuring the desired levels of constraint.

Keywords: Instantaneous helical axis; screw displacement axis; functional axis; wrist 
Akinnola et al., Journal of Biomechanics, accepted 20/10/2020

\section{Introduction}

Understanding the natural axes of a joint is crucial for novel prosthetic and arthroplasty design (Brumbaugh et al., 1982; Stokdijk et al., 2000) and musculoskeletal modelling (Goislard De Monsabert et al., 2018), as the success of these techniques depends on replicating normal joint kinematics. Axes of rotation have been used to validate arthroplasty design (Hooke et al., 2015), and assess joint function (Pothrat et al., 2015), quantify joint instability (Duck et al., 2003), to determine appropriate joint modelling simplifications (Sheehan, 2010), classify joint pathology (Wolf and Degani, 2006), and determine joint centres of rotation with the aim of improving rehabilitation activities and reduce arthroplasty failure (Akhbari et al., 2020; Amabile et al., 2016). Thus, it is vital that the description of the axes and their relationship to each other in vivo is understood.

The wrist joint complex is often modelled as a simple double-hinge joint with non-intersecting axes (Kennedy and Huang, 2016). These axes describe the two main degrees of freedom of the wrist: flexion-extension (FE) and radioulnar deviation (RUD). The relative position and orientation of these axes has previously been investigated, but findings have been inconsistent. Andrews and Youm (1979) measured the FE axis to be orthogonal and approximately $5 \mathrm{~mm}$ proximal to the RUD axis using instantaneous helical axes (IHA). IHAs describe the motion performed as a rotation about and a translation along the axis. Likewise, Leonard et al. (2005) estimated the offset to be $6.8 \mathrm{~mm}$ with the FE axis proximal using a model as opposed to direct measurement. In contrast, Brumbaugh et al. (1982) reported that the axes were almost coincident and not orthogonal. Historically, the axes have been defined in cadaveric studies or, if in vivo, with planar constraints; however, placing hard constraints, 
such as attaching a specimen to a rig with embedded axis of rotation, will certainly prescribe the axis of motion. These will not necessarily represent the axes of motion that occur in vivo. The objective of this study was to investigate the in vivo, 3D relative position and orientation of the natural axes of rotation of the wrist during guided and unguided motions. The primary aim was to determine how guiding the motion affected the axes of rotation in order to determine whether the axes defined in vitro are representative of unguided axes of planar motion. It was hypothesised that the use of a constraint will alter the relative position and orientation of the FE and RUD axes of the wrist. This will have implications for the appropriateness of using in vitro axis definitions for in vivo applications, such as musculoskeletal modelling and wrist arthroplasty development.

\section{Materials and methods}

\subsection{Experimental design}

An eight-camera optical motion capture system (Oqus 500+ cameras, Qualisys, Gothenburg, Sweden) was used to record the wrist motion of 23 healthy participants, 10 male (1.76 \pm 0.07 $\mathrm{m} ; 76.4 \pm 12.9 \mathrm{~kg})$ and 13 female $(1.67 \pm 0.07 \mathrm{~m} ; 62.0 \pm 9.2 \mathrm{~kg})$. Two sets of three $10 \mathrm{~mm}$ diameter reflective markers were arranged in non-linear clusters that were affixed to each participant's dominant hand, specifically over the third metacarpal, and forearm, approximately $2 \mathrm{~cm}$ proximal to the radial and ulnar styloids. Cluster placement was selected to minimise skin motion artefact by avoiding bony prominences (Schmidt et al., 1999). The medial and lateral epicondyles, radial and ulnar styloids, head and base of the third metacarpal, and head of the second metacarpal were digitised and used to create the joint 
coordinate system recommended by the International Society of Biomechanics (Wu et al., 2005).

Participants sat at a table on a stool of adjustable height. Stool height was set so participants could rest their elbow on the table with their shoulder flexed approximately $90^{\circ}$. Each participant performed 15 cycles, in three groups of five continues cycles, of two variants of FE and RUD, one guided (gFE and gRUD) and one unguided (UFE and URUD) (Figure 1). In guided motions, the secondary degree of freedom was constrained, e.g. gFE represented motions where RUD was restricted. Participants were given verbal instruction and a visual demonstration as to how to perform each movement and were requested to use their full range of motion. They were also given the opportunity to practice each movement. Unguided motion was performed with the hand vertically above the elbow and pointing towards the ceiling. Guided motion was performed with the hand and forearm flush against the table, with the elbow positioned at $90^{\circ}$ flexion. UFE, gFE and URUD were performed with the forearm in a neutral position; gRUD was performed with the forearm pronated.
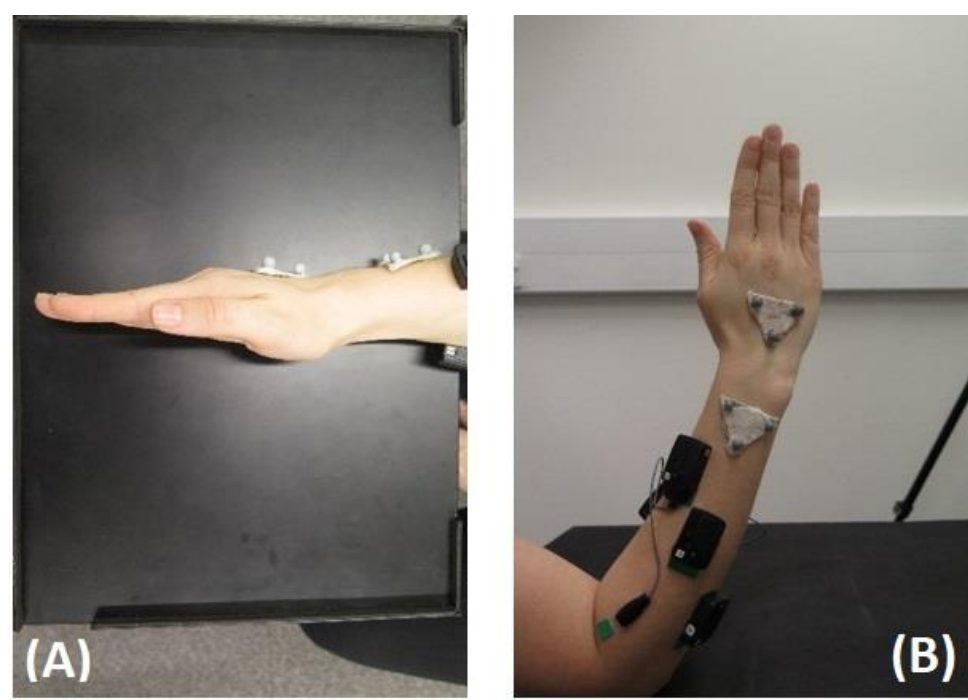

Figure 1: The position and orientation of the hand and forearm during the $(A)$ guided flexion-extension and (B) unguided flexion-extension and radioulnar deviation. The arm was similarly orientated to $(A)$ during guided radioulnar deviation, but the forearm was pronated so that the palm was flush against the surface. 


\subsection{Data processing}

The IHA calculations developed by Woltring et al. (Woltring, 1990, 1991; Woltring et al., 1987) were used to define the axes of rotation for each time step. This method has previously been used to define other natural axes of the upper limb (Hooke et al., 2015; Kennedy and Huang, 2016; Nordin and Frankel, 2001; Ohberg et al., 2003; Stokdijk et al., 2000). A screw displacement axis (SDA), which represents the axis of rotation for a complete motion, was calculated for each cycle. The optimal pivot point of the axis was the mean pivot closest to the IHAs in a least-squared sense (Veeger and Yu, 1996). Similarly, the direction of each axis was calculated as the vector with the smallest angular difference from all the IHAs. A mean SDA was determined for each set of 15 cycles. All calculations were performed using customwritten code in MATLAB (MathWorks, Natick, MA, USA).

\subsection{Data analysis}

The repeatabilities of the motions for each participant were determined by the statistical comparison of the kinematics using SPM one-way ANOVA (MATLAB, MathWorks, Natick, MA, USA). The within-participant variation in the IHAs and ranges of motion were compared with t-tests. The 15 SDAs that represented the axes of the URUD and gRUD motions were compared relative to the axes of the UFE and gFE motions, resulting in four combinations, C1C4 (Table 1). To enable comparison with values from the literature, the offset of each RUD axes with respect to each FE axes was measured in the long axis of the forearm and the angular offset was measured in the plane normal to the long axis of the forearm. To see how range of motion affected the SDAs, the IHAs were grouped by the wrist rotation angle in increasing intervals of $\pm 15^{\circ}$, i.e., $\pm 15^{\circ}, \pm 30^{\circ}, \pm 45^{\circ}$ about neutral; a new representative SDA was calculated for each group. The one-way ANOVA test was used to compare the position 
and angle of the FE and RUD SDAs. Post hoc analysis was conducted with the Tukey Kramer Honest significant difference criterion. The significance level for all tests was 0.05 .

Table 1: Combinations of the guided and unguided variations of flexion-extension (FE) and radioulnar deviation (RUD) screw displacement axes. These combinations have been referred to as C1-C4 within the text.

\begin{tabular}{|c|c|c|c|}
\hline \multicolumn{2}{|c|}{} & \multicolumn{2}{|c|}{ FE } \\
\cline { 3 - 4 } \multicolumn{2}{|c|}{} & Unguided & Guided \\
\hline \multirow{2}{*}{ RUD } & Unguided & C1 & C2 \\
\cline { 2 - 4 } & Guided & C3 & C4 \\
\hline
\end{tabular}

\section{Results}

No differences were found between the cycles of motion; thus, it was concluded that the motions were repeatable. A representative plot of a participant's IHAs during an FE motion and the derived SDA are shown in Figure 2. When comparing the repeatability of both the position and orientation between the IHAs for UFE $\left(1.8 \pm 0.08^{\circ}, 12.3 \pm 2.0 \mathrm{~mm}\right)$ and $\mathrm{gFE}(1.7$ $\pm 0.08^{\circ}, 16.4 \pm 2.9 \mathrm{~mm}$ ) motions, constraining the motion reduced the variation in orientation $(p=0.02)$ but increased the variation in position $(p=0.03)$ for FE (Table 2$)$. Differences were also seen in the repeatability of the IHA orientation between the URUD $\left(1.7 \pm 0.08^{\circ}\right)$ and gRUD $\left(1.5 \pm 0.08^{\circ}\right)$ motions $(p=0.02)$.

Table 2: Mean (standard deviation) within-participant variation in the position and orientation of the instantaneous helical axes for flexion-extension (FE) and radioulnar deviation (RUD).

\begin{tabular}{|l|l|l|}
\hline Motion & $\begin{array}{l}\text { Orientation } \\
\text { repeatability }\left(^{\circ}\right)\end{array}$ & $\begin{array}{l}\text { Positional } \\
\text { repeatability (mm) }\end{array}$ \\
\hline Unguided FE & $1.8(0.08)$ & $12.3(2)$ \\
\hline Guided FE & $1.7(0.08)$ & $16.4(2.9)$ \\
\hline Unguided RUD & $1.7(0.08)$ & $21.6(2.2)$ \\
\hline Guided RUD & $1.5(0.08)$ & $21.4(2.8)$ \\
\hline
\end{tabular}


A

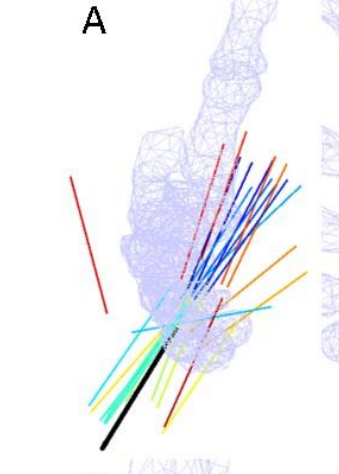

$\mathrm{C}$

Figure 2: A representative plot of the instantaneous helical axes (IHAs) (coloured axes) of the wrist during flexion-extension in the transverse (A), coronal (B), and sagittal (C) planes, as well as an isometric (D) view. The black vector is the screw displacement axis that is derived from the IHAs.

ANOVA testing showed a significant difference in the relative positions of the SDAs $(p=0.03)$. Specifically, the offset of the RUD SDA with respect to the FE SDA in C3 $(0.1 \pm 4.3 \mathrm{~mm})$ differed from C4 (1.6 $\pm 4.0 \mathrm{~mm} ; \mathrm{p}=0.02)$. When comparing the angular difference between the FE and RUD SDAs, differences were again found with ANOVA $(p<0.001)$. Specifically, there were differences between $\mathrm{C} 1\left(53.2 \pm 10.8^{\circ}\right)$ and $\mathrm{C} 4\left(107.8 \pm 17.7^{\circ}\right)$, as well as $\mathrm{C} 2\left(67.5 \pm 14.5^{\circ}\right)$ and C3 $\left(93.5 \pm 16.0^{\circ}\right)(p<0.001)$. Figures 3 and 4 show representative plots of how the SDAs changed as the range of motion was expanded from the neutral pose, i.e., $0^{\circ} \mathrm{FE}$ and RUD.

Guided motions had smaller ranges of motion than unguided motions during both FE $(p<$ 0.001 ) and RUD ( $p<0.001 ;$ Table 3). Differences were found between the orientation of gFE 
and UFE SDAs above the central $\pm 30^{\circ}$ range $(p<0.001)$. There were also differences $(p<0.001)$

between the orientation of gRUD and URUD SDAs for all but the largest range.

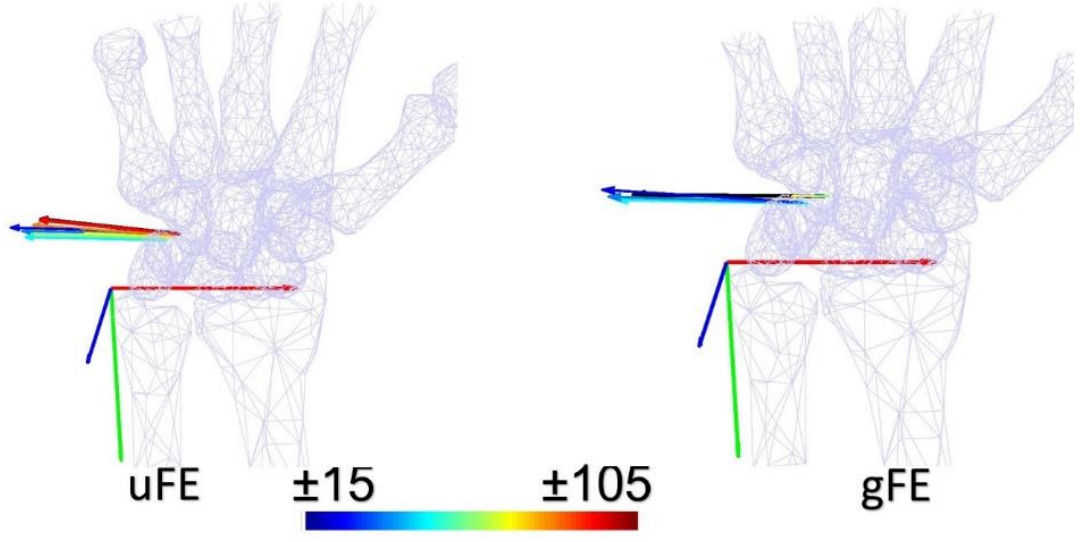

Figure 3: The orientation and position of the unguided ( $\mathrm{UFE}$ ) and guided (gFE) flexionextension screw displacement axes (SDAs) with ranges of motion varying between $\pm 15^{\circ}$ and $\pm 105^{\circ}$ for a single participant. The black vector is the SDA that is derived from the IHAs for the entire range of motion.

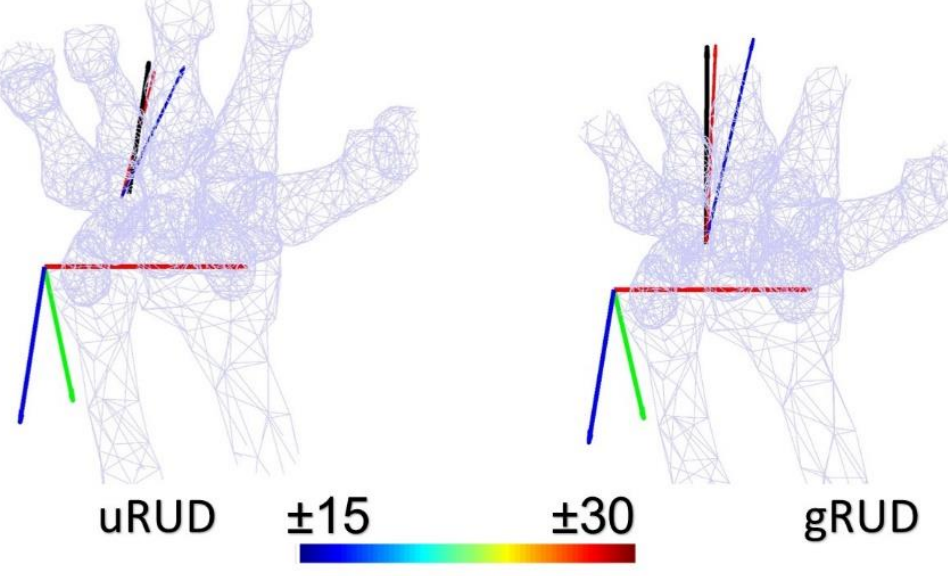

Figure 4: The orientation and position of the unguided (URUD) and guided (gRUD) radioulnar deviation screw displacement axes (SDAs) with ranges of motion varying between $\pm 15^{\circ}$ and $\pm 30^{\circ}$ for a single participant. The black vector is the SDA that is derived from the IHAs for the entire range of motion.

Table 3: Mean ( \pm one standard deviation) range of motion magnitude across all participants in flexion-extension (FE) and radioulnar deviation (RUD).

\begin{tabular}{|l|l|}
\hline Motion & Mean range of motion $\left(^{\circ}\right.$ ) \\
\hline Unguided FE & $137.5(32.8)$ \\
\hline Guided FE & $112.9(35.9)$ \\
\hline Unguided RUD & $64.0(17.2)$ \\
\hline Guided RUD & $43.8(13.0)$ \\
\hline
\end{tabular}


Akinnola et al., Journal of Biomechanics, accepted 20/10/2020

\section{Discussion}

The relative positional and angular offsets between the SDAs for the guided motions were statistically different from those for the unguided motions. Only $\mathrm{C} 1$ and $\mathrm{C} 2$ were not found to be statistically different from each other in either the positional offset or angular difference. This suggests that guiding FE has a greater effect on the relative position and orientation of the axes than guiding RUD. Constraining the plane of RUD does affect the relative orientation of the axes, but only if the FE motion is constrained. When considering how the axes change as the range of motion increased, it was found that the orientation of the FE SDAs differed for ranges of motion greater than $\pm 45^{\circ}$. The RUD SDAs were different for all but the largest range of motion. This implies that guiding the FE motion has a greater effect on the path of the hand as the range of motion increases, whereas both gRUD and URUD motions converge to a similar path as the range of motion increases. This is perhaps due to the mechanical axes of the wrist being oblique to the anatomical axes, in combination with the larger range of motion in FE (Crisco et al., 2011). A participant performing uFE may find it more difficult to determine when the wrist begins to ulnarly deviate, as compared to performing URUD and determining when the wrist begins to flex or extend. Thus, imposing a constraint has a larger effect.

The effect of constraints on the kinematics is corroborated by the variation in the IHAs when averaging them to form the SDA. The orientations of the axes were more uniform when the motion was guided, which was to be expected (Figure 3). While there was no statistical difference in the positional variation between gRUD and URUD, the positional variation of the FE axis was larger, meaning that applying a guide causes the axis to shift. Both findings suggest that natural wrist motion is not planar, which corroborates previous conclusions about wrist motion (Crisco et al., 2011), and that using a guide changes the kinematics of the joint complex. Consequently, musculoskeletal models of healthy wrists should endeavour to use 
Akinnola et al., Journal of Biomechanics, accepted 20/10/2020

axes defined in vivo. However, one reason for investigating the definitions of the wrist is for pathological joints and assessing wrist arthroplasty design, where the axis definitions for a healthy wrist are not available. Further research into the similarities between the axis definitions of the contralateral limb may offer a means of benchmarking kinematics for pathological wrists and investigating similarities in the axes across demographics may allow for axis definitions defined in vivo to be used as objectives in arthroplasty design.

The data presented also elucidate some of the discrepancies in previously published data. C2 produced axes that were similar to the findings of Andrews and Youm (1979) and Leonard et al. (2005), which employed planar rigs, whereas C3 was similar to those axes defined by Brumbaugh et al. (1982), in which participants were asked to perform the motion with a clenched fist. (Salvia et al., 2000) performed uFE and URUD in their study and reported the positions of the axes relative to the capitate so a comparison of the offsets was not possible. However, the reported standard deviations in the positions of the axes were similar to those found in the relative axis positions of this study. The data suggest that the presence of guided or constrained motions, and not physiological differences, is the primary source of differences in the data presented in the literature. It is recommended that researchers define the axes of rotation specifically for their study or select axes from the literature that reflect the same level of constraint to avoid results being affected by unknown or different constraints.

A limitation of this study is that optical motion tracking systems record the trajectory of the reflective markers and not specifically the segments to which they are attached, thus skin motion can affect the derived kinematics. Effort was taken to mitigate this, however; and previous research has shown optical motion tracking to be able to capture kinematics of the wrist accurately (Schmidt et al., 1999). Another limitation of this study is how sensitive IHAs 
are to angular velocity. Part of this study was to investigate how the extremes of motion affected the position and orientation of the axis of rotation for the wrist. However, a minimum angular velocity was used to eliminate low quality IHAs. The extremes of motion feature lower velocity motions due to changes in direction and thus these extreme ranges may be under-represented in the final SDA. Differences in the position and orientation of the SDAs were found throughout the motion, however; so, the conclusions of the study remain reasonable.

\section{Conclusion}

The data presented in this paper show that constraining motion has a significant effect on the definition, both relative position and orientation, of the axes of rotation of the wrist, especially the FE axis. Thus, the use of functional axes of wrist motion derived from in vitro experiments, which often involve kinematic constraints, for in vivo applications, such as musculoskeletal modelling, is likely to be inappropriate. Furthermore, the data suggest that using cadaveric specimens to assess the ability of wrist arthroplasty to maintain joint kinematics may be inappropriate as the axes are likely to differ to those observed in vivo

\section{Conflict of interest statement}

The authors have no conflicts of interest to declare.

\section{References}

Akhbari, B., Morton, A.M., Shah, K.N., Molino, J., Moore, D.C., Weiss, A.-P.C., Wolfe, S.W., Crisco, J.J., 2020. Proximal-distal shift of the center of rotation in a total wrist arthroplasty is more than twice of the healthy wrist. J. Orthop. Res. 38, 1575-1586. 
Amabile, C., Bull, A.M.J., Kedgley, A.E., 2016. The centre of rotation of the shoulder complex and the effect of normalisation. J. Biomech. 49, 1938-1943.

https://doi.org/10.1016/j.jbiomech.2016.03.035

Andrews, J.G., Youm, Y., 1979. A biomechanical investigation of wrist kinematics. J. Biomech. 12, 83-93. https://doi.org/10.1016/0021-9290(79)90012-5

Brumbaugh, R.B., Crowninshield, R.D., Blair, W.F., Andrews, J.G., 1982. An in-vivo study of normal wrist kinematics. J. Biomech. Eng. 104, 176-181. https://doi.org/10.1115/1.3138345

Crisco, J.J., Heard, W.M., Rich, R.R., Paller, D.J., Wolfe, S.W., 2011. The mechanical axes of the wrist are oriented obliquely to the anatomical axes. J. Bone Joint Surg. Am. 93, 169-177. https://doi.org/10.2106/JBJS.I.01222

Duck, T.R., Dunning, C.E., Armstrong, A.D., Johnson, J.A., King, G.J.W., 2003. Application of screw displacement axes to quantify elbow instability. Clin. Biomech. 18, 303-310. https://doi.org/10.1016/S0268-0033(03)00021-4

Goislard De Monsabert, B., Edwards, D., Shah, D., Kedgley, A., 2018. Importance of consistent datasets in musculoskeletal modelling: a study of the hand and wrist. Ann. Biomed. Eng. 46, 71-85. https://doi.org/10.1007/s10439-017-1936-z Hooke, A.W., Pettersson, K., Sagerfors, M., An, K., Rizzo, M., 2015. An Anatomic and Kinematic Analysis of a New Total Wrist Arthroplasty Design. J. Wrist Surg. 4, 121-127. https://doi.org/10.1055/s-0035-1549288 North Am. 47, 207-218. https://doi.org/10.1016/j.ocl.2015.08.018

Leonard, L., Sirkett, D., Mullineux, G., Giddins, G.E.B., Miles, A.W., 2005. Development of an in-vivo method of wrist joint motion analysis. Clin. Biomech. 20, 166-171. https://doi.org/10.1016/j.clinbiomech.2004.09.005

255 Nordin, M., Frankel, V.H., 2001. Biomechanics of the Wrist and Hand, in: Basic Biomechanics of the Musculoskeletal System. Lippincott Williams \& Wilkins. 
Ohberg, F., Grip, H., Wiklund, U., Sterner, Y., Karlsson, J.S., Gerdle, B., 2003. Chronic whiplash associated disorders and neck movement measurements: an instantaneous helical axis approach. IEEE Trans. Inf. Technol. Biomed. 7, 274-282. https://doi.org/10.1109/TITB.2003.821328

Pothrat, C., Goislard de Monsabert, B., Vigouroux, L., Viehweger, E., Berton, E., Rao, G., 2015. Quantifying foot deformation using finite helical angle. J. Biomech. 48, 3716-3719. https://doi.org/10.1016/j.jbiomech.2015.07.044

Salvia, P., Woestyn, L., David, J.H., Feipel, V., Van, S., Jan, S., Klein, P., Rooze, M., 2000.

265 Analysis of helical axes, pivot and envelope in active wrist circumduction. Clin. Biomech. 15, 103-111. https://doi.org/10.1016/S0268-0033(99)00055-8

Schmidt, R., Disselhorst-Klug, C., Silny, J., Rau, G., 1999. A marker-based measurement procedure for unconstrained wrist and elbow motions. J. Biomech. 32, 615-621. https://doi.org/10.1016/S0021-9290(99)00036-6

270 Sheehan, F.T., 2010. The instantaneous helical axis of the subtalar and talocrural joints: a on-invasive in vivo dynamic study. J. Foot Ankle Res. 3, 13-13.

https://doi.org/10.1186/1757-1146-3-13

Stokdijk, M., Biegstraaten, M., Ormel, W., de Boer, Y.A., Veeger, H.E.J., Rozing, P.M., 2000. Determining the optimal flexion-extension axis of the elbow in vivo - a study of 275 interobserver and intraobserver reliability. J. Biomech. 33, 1139-1145. https://doi.org/10.1016/S0021-9290(00)00079-8 Veeger, D., Yu, B., 1996. Orientation of axes in the elbow and forearm for biomechanical modelling, in: Southern Biomedical Engineering Conference - Proceedings. pp. 377-380. https://doi.org/10.1109/SBEC.1996.493254

Woltring, H.J., 1991. Representation and calculation of 3-D joint movement. Hum. Mov. Sci. 10, 603-616. https://doi.org/10.1016/0167-9457(91)90048-3

Woltring, H.J., 1990. Estimation of the trajectory of the instantaneous centre of rotation in planar biokinematics. J. Biomech. 23, 1273-1274. https://doi.org/10.1016/00219290(90)90385-G 
Woltring, H.J., de Lange, A., Kauer, J.M.G., Huiskes, R., 1987. Instantaneous helical axis estimation via natural, cross-validated splines, in: Bergmann, G., Kölbel, R., Rohlmann, A. (Eds.), Biomechanics: Basic and Applied Research: Selected Proceedings of the Fifth Meeting of the European Society of Biomechanics, September 8-10, 1986, Berlin, F.R.G. Springer Netherlands, Dordrecht, pp. 121-128.

Wu, G., van der Helm, F.C.T., (DirkJan) Veeger, H.E.J., Makhsous, M., Van Roy, P., Anglin, C., Nagels, J., Karduna, A.R., McQuade, K., Wang, X., Werner, F.W., Buchholz, B., 2005. ISB recommendation on definitions of joint coordinate systems of various joints for the reporting of human joint motion-Part II: shoulder, elbow, wrist and hand. J. Biomech. 38, 981-992. https://doi.org/10.1016/j.jbiomech.2004.05.042 